\title{
EIGENVALUE PROBLEM MEETS SIERPINSKI TRIANGLE: COMPUTING THE SPECTRUM OF A NON-SELF-ADJOINT RANDOM OPERATOR
}

\author{
Simon N. Chandler-Wilde, Ratchanikorn Chonchaiya \\ AND MARKO LINDNER
}

\begin{abstract}
The purpose of this paper is to prove that the spectrum of the non-self-adjoint oneparticle Hamiltonian proposed by J. Feinberg and A. Zee (Phys. Rev. E 59 (1999), 6433-6443) has interior points. We do this by first recalling that the spectrum of this random operator is the union of the set of $\ell^{\infty}$ eigenvalues of all infinite matrices with the same structure. We then construct an infinite matrix of this structure for which every point of the open unit disk is an $\ell^{\infty}$ eigenvalue, this following from the fact that the components of the eigenvector are polynomials in the spectral parameter whose non-zero coefficients are \pm 1 's, forming the pattern of an infinite discrete Sierpinski triangle.
\end{abstract}

Mathematics subject classification (2010): Primary 47B80; Secondary 47A10, 47B36.

Keywords and phrases: random matrix, spectral theory, Jacobi matrix, disordered systems.

\section{REFERENCES}

[1] W. Amrein, M. Mantoiu and R. Purice, Propagation properties for Schrödinger operators affiliated with certain $C^{*}$-algebras, Ann. H. Poincaré Int 3 (2002), 1215-1232.

[2] A. Böttcher And M. Lindner, Pseudospectrum, Scholarpedia 3, 3 (2008), 2680. http://www.scholarpedia.org/article/Pseudospectrum

[3] A. BötTCher And B. Silbermann, Analysis of Toeplitz Operators, 1st ed., Akademie-Verlag 1989 and Springer 1990; 2nd ed., Springer 2006.

[4] R. Carmona And J. Lacroix, Spectral Theory of Random Schrödinger Operators, Birkhäuser, Boston, 1990.

[5] S. N. Chandler-Wilde, R. Chonchaiya And M. Lindner, Upper Bounds on the Spectra and Pseudospectra of Jacobi and Related Operators, in preparation.

[6] S. N. Chandler-Wilde, R. Chonchaiya And M. Lindner, On the Spectra and Pseudospectra of a Class of non-self-adjoint Random Matrices and Operators, in preparation.

[7] S. N. Chandler-Wilde And M. Lindner, Sufficiency of Favard's condition for a class of banddominated operators on the axis, J. Funct. Anal. 254 (2008), 1146-1159.

[8] S. N. Chandler-Wilde And M. Lindner, Limit Operators, Collective Compactness, and the Spectral Theory of Infinite Matrices, Memoirs of the American Mathematical Society, Vol. 210, Nr. 989, 2011.

[9] G. M. Cicuta, M. Contedini And L. Molinari, Non-Hermitian tridiagonal random matrices and returns to the origin of a random walkn, J. Stat. Phys. 98 (2000), 685-699.

[10] E. B. DAVIES, Spectral properties of non-self-adjoint matrices and operators, Proc. Royal Soc. A. 457 (2001), 191-206.

[11] E. B. DAVIES, Spectral theory of pseudo-ergodic operators, Commun. Math. Phys. 216 (2001), 687704.

[12] E. B. DaviES, Linear Operators and their Spectra, Cambridge University Press, 2007.

[13] D. Doty, X. Gu, J. H. Lutz, E. Mayordomo And P. Moser, Zeta dimension, in Mathematical Foundations of Computer Science 2005, Springer, 2005, pp. 283-294.

[14] K. J. Falconer, Fractal Geometry: Mathematical Foundations \& Applications, John Wiley \& Sons, 1990. 
[15] J. Feinberg And A. ZeE, Non-Hermitean Localization and De-Localization, Phys. Rev. E 59 (1999), 6433-6443.

[16] J. Feinberg and A. ZeE, Spectral Curves of Non-Hermitean Hamiltonians, Nucl. Phys. B 552 (1999), 599-623.

[17] V. GeOrgescu And S. Golenia, Isometries, Fock spaces and spectral analysis of Schrödinger operators on trees, Journal of Functional Analysis 227 (2005), 389-429.

[18] V. Georgescu And A. Iftimovici, $C^{*}$-Algebras of Energy Observables: I. General Theory and Bumps Algebras, Preprint 00-521 at http://www .ma .utexas . edu/mp_arc/, December 2000.

[19] V. Georgescu And A. Iftimovici, Crossed products of $C^{*}$-algebras and spectral analysis of quantum Hamiltonians, Comm. Math. Phys. 228 (2002), 519-560.

[20] V. Georgescu AND A. IfTImovici, Localization at infinity and essential spectrum of quantum Hamiltonians, Rev. Math. Phys. 18 (2006), 417-483.

[21] I. GOLdSHEID AND B. KhORUZHENKo, Eigenvalue curves of asymmetric tridiagonal random matrices, Electronic Journal of Probability 5 (2000), 1-28.

[22] D.E. Holz, H. ORland ANd A. ZeE, On the remarkable spectrum of a non-Hermitian random matrix model, Journal of Physics A: Mathematical and General 36 (2003), 3385-3400.

[23] V. G. Kurbatov, Functional Differential Operators and Equations, Kluwer Academic Publishers, Dordrecht, Boston, London, 1999.

[24] J. I. Lathropa, J. H.LuTZ, AND S. M. Summers, Strict self-assembly of discrete Sierpinski triangles, Theor. Computer Sci. 410 (2009), 384-405.

[25] Y. LAST AND B. Simon, Eigenfunctions, transfer matrices, and absolutely continuous spectrum of one-dimensional Schrödinger operators, Invent. Math. 135 (1999), 329-367.

[26] Y. LAST AND B. Simon, The essential spectrum of Schrödinger, Jacobi and CMV operators, J. Anal. Math. 98 (2006), 183-220.

[27] M. Lindner, Infinite Matrices and their Finite Sections: An Introduction to the Limit Operator Method, Frontiers in Mathematics, Birkhäuser 2006.

[28] M. Lindner, Fredholmness and index of operators in the Wiener algebra are independent of the underlying space, Operators and Matrices 2 (2008), 297-306.

[29] M. LindNER, A note on the spectrum of bi-infinite bi-diagonal random matrices, Journal of Analysis and Applications 7 (2009), 269-278.

[30] M. Lindner, Fredholm Theory and Stable Approximation of Band Operators and Generalisations, Habilitation thesis, TU Chemnitz, 2009.

[31] M. Mantoiu, $C^{*}$-algebras, dynamical systems at infinity and the essential spectrum of generalized Schrödinger operators, J. Reine Angew. Math. 550 (2002), 211-229.

[32] L. A. Pastur and A. L. Figotin, Spectra of Random and Almost-Periodic Operators, Springer, Berlin, 1992.

[33] V. S. Rabinovich And S. Roch, The essential spectrum of Schrödinger operators on lattices, J. Phys. A: Math. Gen. 39 (2006), 8377-9394.

[34] V. S. Rabinovich, S. Roch And B. Silbermann, Fredholm Theory and Finite Section Method for Band-dominated operators, Integral Equations Operator Theory 30, 4 (1998), 452-495.

[35] V. S. Rabinovich, S. Roch and B. Silbermann, Limit Operators and Their Applications in Operator Theory, Birkhäuser, 2004.

[36] C. RemLing, The absolutely continuous spectrum of Jacobi matrices, arXiv:0706.1101v1, June 2007.

[37] C. Remling, The Absolutely Continuous Spectrum of One-dimensional Schrödinger Operators, Math. Phys., Anal. and Geom. 10 (2007), 359-373.

[38] L. N. TRefethen And M. Embree, Spectra and pseudospectra: The behavior of nonnormal matrices and operators, Princeton University Press, Princeton, NJ, 2005. 